Planetary Systems in the Universe - Observation, Formation and Evolution

Proceedings IAU Symposium No. 202, (C)2004 IAU

Alan Penny, Pawel Artymowicz, Anne-Marie Lagrange, \& Sara Russell, eds.

\title{
A scattered Uranus and Neptune, and implications for the asteroid belt
}

\author{
Edward W. Thommes, Martin J. Duncan \\ Queen's University, Kingston, Ontario, Canada
}

Harold F. Levison

Southwest Research Institute, Boulder, CO, USA

John E. Chambers

NASA Ames, Moffat Field, CA, USA

\begin{abstract}
It has been proposed that Uranus and Neptune originated interior to $\sim 10 A U$, as potential gas giant cores which were scattered outward when Jupiter won the race to reach runaway gas accretion. We present further numerical simulations of this scenario, which show that it reproduces the present configuration of the outer Solar System with a high degree of success for a wide range of initial conditions. Also, we show that this mechanism may have simultaneously ejected planets from the asteroid belt.
\end{abstract}

\section{Introduction}

Analytic estimates (eg. Kokubo and Ida 2000) and numerical simulations (eg. Davis, Farinella and Weidenschilling 1999) show that it is difficult to understand the formation of Uranus and Neptune in the lifetime of the Solar System, to say nothing of the lifetime of the gas nebula ( $\sim 10 \mathrm{Myrs})$ from which the outer giant planets each accreted $\sim 1-2 \mathrm{M}_{\oplus}$ of hydrogen and helium. Numerical simulations by Brunini and Fernandez (1999) produce Uranus- and Neptune-mass bodies in a few $10^{7}$ years, but these simulations neglect gravitational interactions among bodies with masses below $1 \mathrm{M}_{\oplus}$; with all interactions included, such rapid formation is only possible if the physical radii of planetesimals are increased by a factor of about ten relative to bodies with a plausible density (Brunini, private communication).

An alternative scenario for the origin of Uranus and Neptune is developed by Thommes, Duncan and Levison (1999): Oligarchic growth (Kokubo and Ida 1998, 2000) initially produces four or more bodies of giant planet core size in what later becomes the Jupiter-Saturn region $(\sim 5-10 \mathrm{AU})$. When one of these acquires a massive envelope through runaway gas accretion (Pollack et al 1996) to become Jupiter, the remaining protoplanets are scattered onto eccentric orbits. However, in crossing the region beyond $\sim 10 \mathrm{AU}$, where most of the mass is still in small planetesimals, the protoplanets experience dynamical friction which decreases their eccentricities and decouples them from Jupiter and each 
other. The innermost scattered protoplanet subsequently also accretes a massive gas envelope and becomes Saturn; the outer two become Uranus and Neptune.

\section{Simulations}

New simulations of this model have been performed, again using the SyMBA integrator (Duncan, Levison and Lee 1998). The surface density of planetesimals used was $10(a / 5 \mathrm{AU})^{-2} \mathrm{~g} / \mathrm{cm}^{2}$, steeper than the profiles used by Thommes, Duncan and Levison (1999), $\sigma \propto a^{-1}$ and $a^{-3 / 2}$. Between $\sim 5-12 \mathrm{AU}$, most of the disk is in the form of $10 \mathrm{M}_{\oplus}$ protoplanets. In the first set of eight runs, there are initially four protoplanets. The mass of the innermost is increased to Jupiter's mass over $10^{5}$ years to simulate runaway gas accretion. The states of the runs after 5 (or 10) Myrs are shown in the left panel of Fig. 1. Most protoplanets have acquired low eccentricities. Five of the runs (A1, A4, A6, A7, and A8) have ended up looking qualitatively similar to the outer Solar System. Of these, A1, A6 and A8 have the outer two protoplanets within $15 \%$ of Uranus and Neptune's semimajor axes. The extent of excitation of the Kuiper belt region in these runs is determined by the furthest aphelion distance of the protoplanets during scattering, giving rise to a "fossilized scattered disk" (Thommes et al 1999). Another set of eight runs is performed with an extra protoplanet (protoJupiter + 4). After $10-15$ Myrs, eccentricities are uniformly low. In two of the eight runs, one $10 \mathrm{M}_{\oplus}$ body has been ejected, leaving the right number of protoplanets (3) beyond Jupiter. In both cases, the outer bodies are within $15 \%$ of Uranus and Neptune's semimajor axes.

\section{Effects on the asteroid belt}

In the above runs, protoplanets often cross the region interior to Jupiter for brief periods of time ( $10^{4}$ years or less) during the initial scattering. The present-day asteroid belt displays an anomalously high degree of excitation and mass depletion (eg. Petit, Morbidelli and Valsecchi 1999); could scattered protoplanets have played a role in this? Other simulations are performed, this time placing additional planetesimals in the asteroid belt region. It is found that scattered protoplanets can excite eccentricities in the outer belt to values comparable to the present ones, but eccentricities in the inner belt, and inclinations everywhere, are not significantly affected. However, scattered protoplanets may have played a more indirect role in sculpting the asteroid belt. The formation of planet-sized bodies in the asteroid belt would have depleted this region of planetesimals, and on Myr timescales they would have raised high eccentricities and inclinations in the remaining planetesimals. Of course these bodies must ultimately have been removed, and this is where scattered protoplanets may come in. Fig. 1 (right) shows the evolution of a system where a $1 \mathrm{M}_{\oplus}$ body has been added in the asteroid belt. At $4 \times 10^{4}$ years, a belt-crossing protoplanet ejects the body. Out of a series of eight such runs, three result in the removal of the $1 \mathrm{M}_{\oplus}$ body. In one of these, though, the protoplanet ends up in the asteroid belt instead. 


\section{Summary}

The scattered protoplanet model readily reproduces the outer Solar System, even with a relatively steep initial planetesimal surface density profile, $\sigma \propto a^{-2}$, and with an "extra" protoplanet. Giant protoplanets crossing the asteroid belt as they are scattered are able to remove $\sim 1 \mathrm{M}_{\oplus}$ bodies; this may have helped to leave the region in its present dynamically excited and mass-depleted state. Another possible outcome is the deposition of a giant protoplanet in the belt. As a way of delivering giant planet core-sized bodies interior to their original formation site, such scattering events may have played a role in the evolution of some of the observed extrasolar planetary systems.
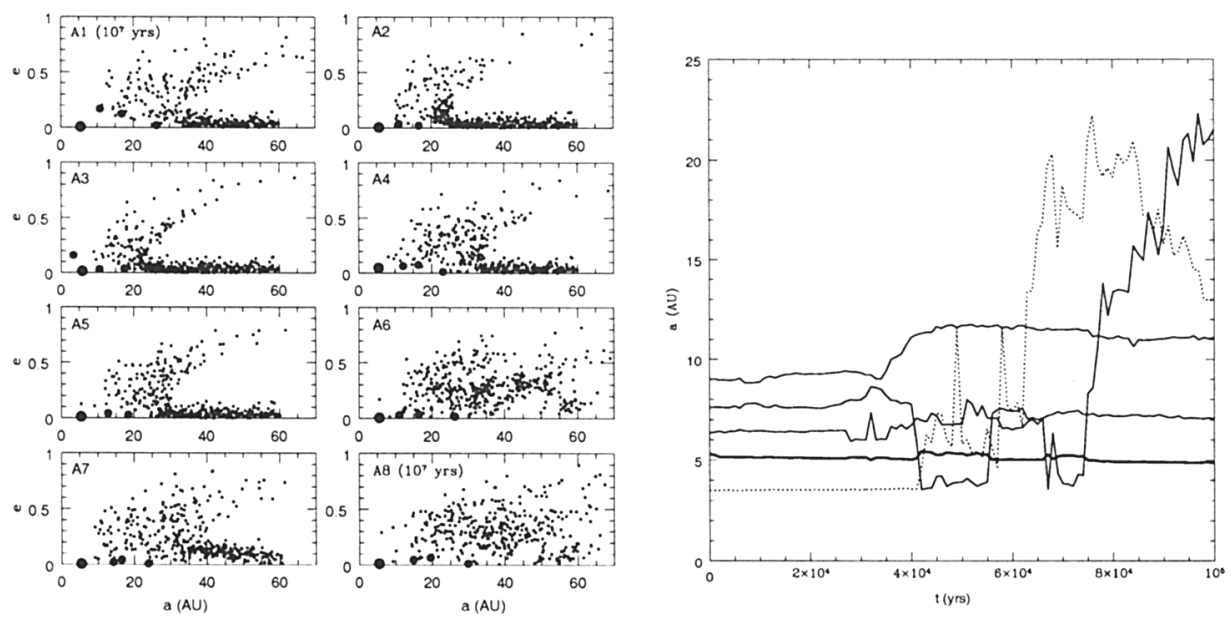

Figure 1. Left: State of Runs A1-A8 at 5 (or 10) Myrs, plotting semimajor axis versus eccentricity. The large, medium and small circles denote Jupiter, $10 \mathrm{M}_{\oplus}$ protoplanets and planetesimals respectively. Right: Evolution of the semimajor axis of Jupiter (thick), three protoplanets (thin) and a $1 \mathrm{M}_{\oplus}$ belt planet (dotted, initially innermost), which is ejected from the belt region at $4 \times 10^{4}$ years.

\section{References}

Brunini, A., \& Fernandez, J. A. 1990, P\&SS, 47, 591

Davis, D. R., Farinella, P., \& Weidenschilling, S. J. 1999, in 30th Lunar and Planetary Science Conference, abstract 1883

Duncan, M. J., Levison, H. F., \& Lee, M.-H. 1998, AJ, 116, 2067

Kokubo, E., \& Ida, S. 1998, Icarus, 131, 171

Kokubo, E., \& Ida, S. 2000, Icarus, 143, 15

Petit, J.-M., Morbidelli, A., \& Valsecchi, G. B. 1999, Icarus, 141, 367

Pollack, J. B. et al 1996, Icarus, 124, 62

Thommes, E. W., Duncan, M. J. \& Levison, H. F. 1999, Nature, 402, 635 